\title{
$\mathrm{PDM}$ 데이터베이스로부터 핵심성과지표를 추출하기 위한 정보 시스템 아키텍쳐
}

\author{
도 남 철 ${ }^{\dagger}$
}

경상대학교 산업시스템공학부, 공학연구원

\section{An Information System Architecture for Extracting Key Performance Indicators from PDM Databases}

\author{
Namchul Do \\ Dep. of Industrial and Systems Engineering, Gyeongsang National University, ERI
}

\begin{abstract}
The current manufacturers have generated tremendous amount of digitized product data to efficiently share and exchange it with other stakeholders or various software systems for product development. The digitized product data is a valuable asset for manufacturers, and has a potential to support high level strategic decision makings needed at many stages in product development. However, the lack of studies on extraction of key performance indicators(KPIs) from product data management(PDM) databases has prohibited manufacturers to use the product data to support the decision makings. Therefore this paper examines a possibility of an architecture that supports KPIs for evaluation of product development performances, by applying multidimensional product data model and on-line analytic processing(OLAP) to operational databases of product data management. To validate the architecture, the paper provides a prototype product data management system and OLAP applications that implement the multidimensional product data model and analytic processing.
\end{abstract}

Keywords: Multidimensional Data Model, On-Line Analytic Processing(OLAP), Product Data Management (PDM), Product Development Performance Evaluation, Key Performance Indicator(KPI)

\section{1. 서 론}

기업들은 1970년대 CAD 시스템 도입을 시작으로 일관성 유지 와 설계 공유를 위하여 제품설계 자료를 디지털 형태로 전환 해 왔다. 현재 대부분의 기업은 통합되고 전산화된 정보 시스 템을 사용하여 방대한 양의 디지털화된 제품설계 자료를 관리 하고 있다. 오랜 기간 동안 축적된 디지털 제품자료는 기업의 귀중한 자산이며, 제품개발과 설계에 관련된 다양한 의사결정 을 지원할 수 있다. 하지만 제품자료로부터 관련 핵심지표의 추출에 대한 연구 부족으로 제품자료 기반 의사결정이 체계적 으로 지원되지 않고 있다.

제품개발 성과평가(Evaluation of Product Development Perfor- mance)는 제품개발이 효과적이고 정상적으로 이루어지고 있는 지를 다양한 지표를 통하여 평가하는 활동이다. 제품개발 성 과평가는 성공적 제품개발을 위한 필수적인 관리 활동이지만, 현재 평가방식은 해당 단계가 끝난 후 추가적인 처리 과정을 통해 지표를 제공하므로 제품개발 진행을 조정하거나 과정 중 에 발생하는 위험에 대응하기 쉽지 않다. 그러므로 현재 제품 개발에 대한 객관적이고 수치적인 평가지표를 실시간 제공할 수 있다면, 제품개발 성과평가와 관리에 큰 도움이 될 것이다.

본 논문에서는 현재 제품개발을 광범위하게 지원하고 있는 제품자료관리(Product Data Management : PDM) 데이터베이스 를 기반으로 제품개발 성과평가를 위한 핵심성과지표(Key Performance Indicator : KPI)의 실시간 추출 방법을 제안한다. 이 방

† 연락저자 : 도남철, 660-701 경남 진주시 진주대로 501 경상대학교 산업시스템공학부, ERI, Tel : 055-772-1703, Fax : 055-772-1699,

E-mail : dnc@gnu.ac.kr

2012년 8월 9일 접수; 2012년 11월 11일 수정본 접수; 2012년 12월 4일 게재 확정. 
법은 다차원 제품자료 모델(Multidimensional Product Data Model) 과 온라인 분석처리(On-Line Analytic Processing : OLAP)를 적 용하여 제품자료를 관리하는 PDM 데이터베이스에서 제품개 발 평가를 위한 핵심성과지표를 실시간 추출한다. 제안된 방 법은 구현될 정보시스템을 고려하여 정보시스템 아키텍처 형 태로 표현된다.

본 연구에서는 제안된 아키텍처를 개발하기 위하여 일반적 인 PDM 데이터베이스 모델을 정의하고, 문헌 조사를 통해 정 리된 지표 중 $\mathrm{PDM}$ 데이터베이스로 부터 실시간으로 추출 할 수 있는 핵심성과지표를 결정한다. 다음으로 기존 PDM 데이 터베이스의 제품정보를 처리하여 실시간 성과지표를 생성하 고, 이를 가공하여 제품개발에 관련된 의사결정을 지원하는 시 스템 아키텍처를 개발한다. 마지막으로 해당 아키텍처를 구현 한 시제품을 개발하여 제안된 모델이 유효함을 확인한다.

본 논문은 다음과 같이 구성되어 있다. 제 2장에서는 관련 연 구를 서술한다. 제 3장에서는 다차원 자료모델(Multidimensional Data Model)과 OLAP 개념을 소개한다. 제 4장에서는 제안 된 아키텍처와 그 구성요소를 설명한다. 제 5장에서는 시제품 $\mathrm{PDM}$ 시스템을 통한 아키텍처 구현과 이 시스템을 활용한 예 를 설명한다. 제 6장에서는 결론을 도출하고 추후 연구 과제를 서술한다.

\section{2. 관련 연구}

본 연구 주제와 관련된 기존 연구는 내용적 측면에서 제품개 발 성과평가와 방법적 측면에서 데이터베이스 분석(Database Analytics) 분야로 구분하여 살펴볼 수 있다. <Figure 1>은 두 가 지 측면의 관련 분야와 연구 대상을 보여주고 있다.

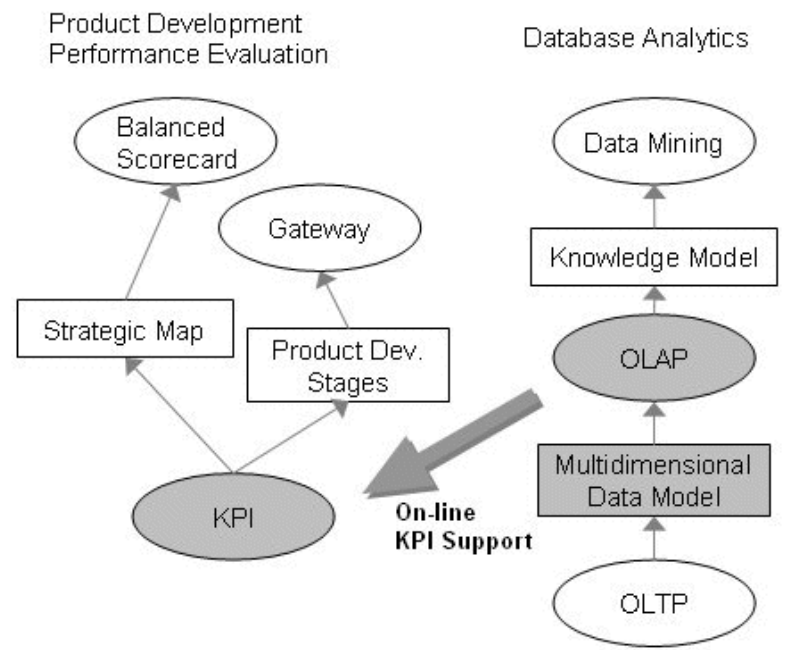

Figure 1. Research domain in product development performance evaluation and database analytics

제품개발 성과평가에서 본 연구의 대상 분야는 제품개발에
대한 핵심성과지표 도출이다. 핵심성과지표(Key Performance Indicator)란 기업의 미래성과에 영향을 주는 여러 핵심지표를 묶은 평가기준을 말한다. 제품개발 성과평가 분야에도 다양한 핵심성과지표가 제안되었으나(Tatikonda, 2007; Cheng et al., 2011; Tan and Rasli, 2011), 본 논문에서 다룬 PDM 데이터베이 스로 부터 핵심성과지표를 추출하는 연구는 소수가 존재한다 (Alemanni et al., 2008; Oh and Yang, 2010). 핵심성과지표를 기 반으로 재무, 내부 프로세스, 인적자원, 그리고 고객 등의 기업 전체 경영을 고려한 Balanced Scorecard(BSC) 방법을 제품개발 에 적용한 연구(Oh and Yang, 2010) 와 핵심성과지표를 기반으 로 제품개발 프로세스에 몇 개의 주요 Gap 분석 과정을 두어 제품개발을 평가하는 Gateway 방법론(MAS-VM, 2012)이 핵심 성과지표에 관한 확대된 연구로 분류될 수 있다 $(<$ Figure $1>$ 의 $\mathrm{BSC}, \mathrm{Gateway}$ 참조). BSC의 경우 핵심성과지표에 Strategic Map 모델을 적용하여 확장하였으며, Gateway의 경우 Product Development Stages 모델을 적용하여 확장하였다(<Figure 1>의 Strategic Map, Product Development Stages 참조).

방법적인 측면에서 본 연구는 데이터베이스 분석(Database Analytics) 중의 OLAP 적용과 관련 있다. PDM 시스템은 제품 설계정보를 대용량 데이터베이스를 통하여 관리하는 데이터 베이스 응용 시스템으로 볼 수 있다. PDM 데이터베이스는 대 량의 복잡한 제품설계 자료를 관리하기 위하여 대용량 자료 처리에 적합한 On-Line Transaction Processing(OLTP)에 기반하 고 있다(<Figure 1>의 OLTP 참조). 본 연구는 의사결정 지원 자 료처리에 적합한 On-Line Analytical Processing(OLAP) 방법을 이용하여 OLTP 기반의 PDM 데이터베이스에서 의사결정을 지원하는 핵심성과지표를 추출한다. 이 때 다차원 자료모델을 사용하며 PDM 데이터베이스의 자료를 의사결정 지원 자료로 변환한다 $(<$ Figure 1>의 OLAP와 Multidimensional Data Model 참고). 그러므로 본 연구는 제품정보 분석용 다차원 자료모델 인 다차원 제품자료모델(Multidimensional Product Data Model) 을 개발하여 PDM 데이터베이스 기반의 제품개발 성과평가를 위한 OLAP를 적용하였다. PDM 데이터베이스를 기반으로 OLAP를 적용한 연구는 아직까지 보고되지 않고 있다.

앞에서 언급한 PDM 기반 제품개발 성과평가 연구(Alemanni et al., 2008; Oh and Yang, 2010)는 PDM에서 핵심성과지표를 추출하기 위하여 OLTP를 이용한다. 이 경우 의사결정을 위한 체계적인 방법인 OLAP 기반 방법에 비하여 유연하지 못하고 복잡한 자료 추출과 변환 과정이 필요하므로, 많은 자원이 소 요되며, 일관되지 못한 자료를 제공할 수 있다.

데이터베이스 분석에서 OLAP보다 발전한 방법이 규칙 자 체를 찾아내는 데이터마이닝(Data Mining)이다(<Figure 1>의 Data Mining 참조). 데이터마이닝을 제품개발에 적용한 연구들 로써는 PDM 데이터베이스와 독립된 단순 제품 개념 설계에 적용한 연구(Yan et al., 2009)와 PDM에서 자연언어처리를 포함 한 검색기능 강화를 위한 연구(Ahmed, 2009, 2011) 등이 존재 한다. 그러나 이 연구들은 PDM 데이터베이스에 축적된 제품 
설계 자료 기반의 의사결정 지원이나 규칙발견을 지원하지 못 하고 있다.

그러므로 본 연구는 제품개발 성과평가를 위한 핵심성과지 표를 다차원 제품자료모델과 온라인 분석처리를 통해 실시간 제공한다는 의미가 있다(<Figure 1>의 화살표 On-line KPI support 참조). 즉, 본 연구는 데이터베이스 분석 기법인 OLAP를 제품개발 성과평가 분야의 핵심성과지표 추출에 적용한 연구 이며, 이를 위해 다차원 제품자료모델과 이를 통한 PDM 데이 터베이스로부터의 분석자료 추출 방법을 제안하였다. <Figure $1>$ 에서는 본 논문의 연구 대상과 상호관계가 색칠한 프로세스 와 모델 그리고 인터페이스로 표시되어 있다(<Figure 1>의 KPI, OLAP, Multidimensional Data Model, 그리고 On-line KPI support 참조).

\section{OLAP와 다차원 자료모델}

\subsection{OLAP}

데이터베이스 관리시스템(Data Base Management System: $\mathrm{DBMS})$ 은 사용자 정의 자료구조를 지원하고, 다수의 사용자와 응용프로그램이 일관성 있는 대용량 자료를 공유할 수 있게 함으로써 컴퓨터 응용 정보시스템의 핵심 기반을 제공하고 있 다. 그러므로 초기의 데이터베이스 기술은 대용량 자료를 신 속하게 읽고, 쓰고, 처리하는 On-Line Transaction Processing (OLTP) 기능에 중심을 두고 있었다.

정보기술의 발전으로 데이터베이스에서 처리하는 대용량 자료를 보다 고급의 의사결정지원에 사용할 수 있는 On-Line Analytic Processing(OLAP) 기술이 나타났다. OLAP란 기존 OLTP와 다른 차원의 추상화를 위하여 대상 자료를 처리하는 것을 뜻한다(Han and Kamber, 2001). 예로 판매 자료를 관리하 는 판매 데이터베이스는 판매된 제품의 수량과 가격을 저장하 고 관리하기 위한 OLTP 기능에 초점이 맞추어져 있다. 하지만 축적된 판매 자료를 바탕으로 계절별 판매 제품과 판매량을 분석하려고 할 경우, 이는 기존 데이터베이스 목적과 다른 차 원의 추상화(계절별 판매 분석)를 목적으로 하는 데이터베이 스 응용이 필요하다. 이와 같이 의사결정을 위한 데이터베이 스 자료를 추출하기 위해서는 OLTP와 다른 자료처리(OLAP) 가 필요하며, 이를 지원하기 위해서는 OLTP 용 데이터베이스 구조를 의사결정 지원에 적절한 자료구조로 바꾸어주는 다차 원 자료모델(Multidimensional Data Model)이 필요하다.

\section{2 다차원 자료모델}

다차원 자료모델(Multidimensional Data Model)은 OLAP 실 행을 위한 기반을 제공하는 자료모델이다. 관계형 데이터베이 스 관리시스템을 사용할 경우 OLTP를 구현하기 위한 자료모 델은 관계형 테이블과 테이블 사이의 관계로 표현되지만, 다
차원 모델의 경우 데이터 큐브(Data Cube) 형태의 모델을 사용 한다. 데이터 큐브는 여러 차원에서 자료에 접근할 수 있도록 차원(Dimension)과 사실(Fact)로 구성된 모델을 정의한다.

차원(Dimension)은 대상에 대한 분석하고 싶은 관점 혹은 특 성을 뜻한다. 예로 판매에 관련된 다차원 자료모델을 만들 경 우, 판매에 관련된 판매시간, 판매자, 판매물품, 그리고 판매장 소 등이 차원이 될 수 있다. 사실(Fact)은 다차원 모델의 중심이 되는 주제를 뜻하며, 이 주제를 중심으로 다수의 차원이 연결 된 형태를 취한다. 판매에 관련된 다차원 모델의 경우 판매 (Sale) 발생이 사실이 될 수 있다.

다차원 자료모델의 구현은 관계형 데이터베이스 모델을 기 반으로 할 경우 스타 스키마(Star Schema)를 따르는 경우가 많 다. 즉 차원 테이블(Dimension Table)과 연결된 사실 테이블 (Fact Table)을 생성한 후, 차원 테이블에 분석하고자하는 자료 를 입력하여 배열하는 것이다. <Figure $2>$ 에는 예의 판매 다차 원 자료모델이 그림으로 표시되어 있다.

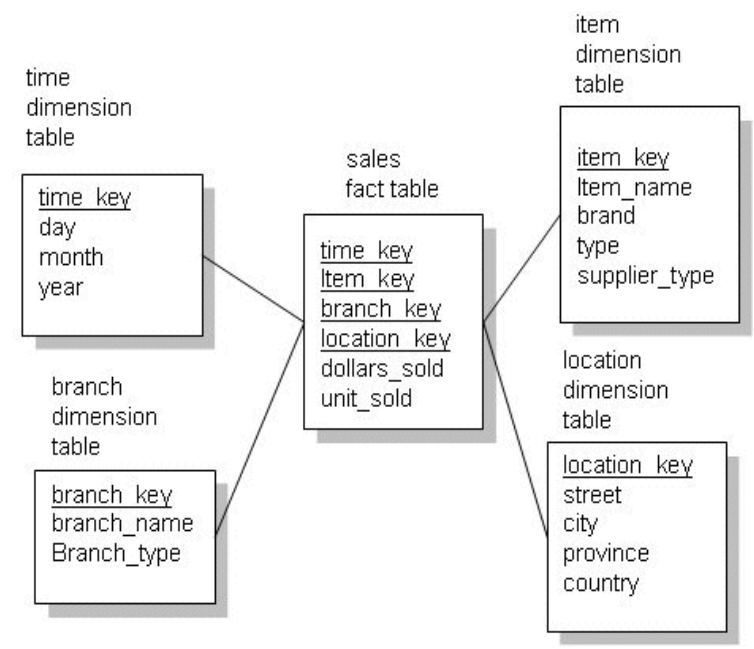

Figure 2. Star schema for implementing sales multidimensional data model(Han and Kamber, 2001)

다차원 모델의 분석이란 사실을 중심으로 각 차원들 간의 관계를 찾아내는 것이다. 예로 판매 다차원 모델의 경우 오전 10 시에(판매시간 차원) 가장 많이 팔린 제품(판매제품 차원)을 찾아내는 것은 판매 사실을 중심으로 판매시간과 판매제품 차 원의 관계를 밝히는 것이다.

\section{3 다차원 제품자료모델}

본 논문에서 제안한 다차원 제품자료 모델(Multidimensional Product Data Model)은 PDM 데이터베이스를 기반으로 데이터 베이스 분석을 지원하는 제품 중심의 다차원 자료모델이다. 데이터베이스 기술 관점에서 제품설계를 지원하는 Product Data Management(PDM)는 대용량 제품설계 정보를 관리하는 일종의 데이터베이스 응용으로 볼 수 있다. $\mathrm{PDM}$ 에 사용하는 
데이터베이스의 목적은 설계대상 제품을 표현하고 관련 대용 량 설계 정보를 빠르게 처리하여 제품개발을 효율적으로 진행 하도록 하는 것이다. 반면에 제품개발 성능평가는 설계과정을 시간, 비용, 그리고 품질 등의 특정 가치 기준을 통하여 평가하 는 목적을 가지고 있다. 그러므로 제품개발 평가를 위한 OLAP 는 제품설계를 효과적으로 실행하기 위하여 광범위하게 축적 되는 제품설계 정보를 제품개발 평가라는 다른 목적을 위하여 자료를 처리하는 과정으로 볼 수 있으며, 이를 위한 다차원 자 료모델이 다차원 제품자료모델이다. 다차원 제품자료모델에 대한 내용은 제 4.3절에서 상세히 다룬다.

\section{4. 제품개발 성과평가 아키텍처}

제품개발 성과평가(Product Development Performance Evaluation: PDPE) 시스템은 기존의 PDM 데이터베이스에 OLAP를 적용하여 실시간으로 제품개발 핵심성과지표를 제공하는 시 스템이다. <Figure 3>는 제안된 PDPE 시스템 아키텍처를 보여 주고 있다. 아키텍처는 기존의 PDM 시스템에 제품개발 성과 평가를 위한 요소를 확장시킨 형태를 띄고 있다. 아키텍처의 PDM 쪽 구성요소는 PDM 데이터베이스, PDM 데이터베이스 모델인 제품자료모델(Product Data Model), 그리고 PDM 클라 이언트(PDM Client)로 구성되어 있다(<Figure 3>의 구성요소 참조).

제품개발 성과평가 부분은 PDM 데이터베이스와 제품자료 모델을 공유하고 있으며, PDPE KPI, PDPE Clients, PDPE Data Warehouse, Extract Transform Load Module(ETL Module) 그리고 다차원 제품자료모델(Multidimensional Product Data Model)로 구성되어있다(<Figure 3>의 구성요소 참조). 이 장에서는 PDPE 시스템 아키텍처의 주요 구성요소를 상세히 설명한다.

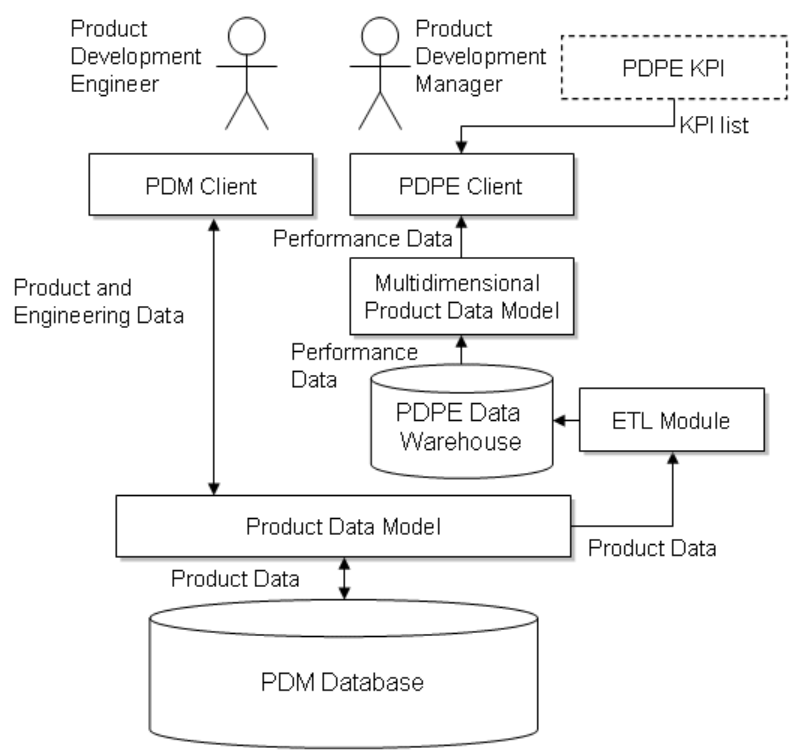

Figure 3. PDPE system architecture

\subsection{PDM 시스템}

$\mathrm{PDM}$ 시스템은 기업의 제품개발 자료와 프로세스를 관리하 는 기간 정보시스템이다. PDM 시스템은 독립적으로 사용되기 도 하지만 최근에는 보다 확장된 Product Life cycle Manage$\operatorname{ment}(\mathrm{PLM})$ 시스템의 핵심 구성 요소로써 사용되기도 한다(Do, 2007). PDM 시스템은 일종의 데이터베이스 응용 시스템이므 로 PDM 클라이언트, PDM 데이터베이스, 그리고 제품자료모 델(PDM 데이터베이스 모델)로 나누어 볼 수 있다.

PDM 클라이언트 $<$ Figure 4>의 PDM Client)는 제품개발 기 술자가 제품개발 과정에서 발생하는 제품정보를 생성, 저장, 그 리고 관리하도록 한다. PDM 클라이언트가 제품개발을 지원하 기 위하여 제공하는 기본 기능은 부품리스트, 기술문서, 제품 구조, 설계변경, 그리고 과제 관리이다.

$\mathrm{PDM}$ 데이터베이스는 제품자료모델에 의거하여 실제 제품 자료를 저장하는 기능을 한다. PDM 데이터베이스에 저장되는 자료는 데이터베이스 레코드 형태로 정의되는 자료와 $\mathrm{CAD}$ 모델 등을 구성하는 이진 자료(Binary Data)로 구분할 수 있다. 레코 드 형태로 저장되는 자료에는 제품 자료 외에 생성일, 생성자 등 의 관리 자료가 포함된다. 본 연구에서 관심을 가지는 데이터는 레코드 형태로 저장된 제품 메타 자료(Product Meta Data)이다.

제품자료모델은 제품 설계 정보가 어떻게 PDM 데이터베이 스에 저장되고 관리되는지를 표현한다. 뒤에서 소개할 다차원 제품자료모델도 제품자료모델을 기반으로 개발되므로, 본 논 문에서는 <Figure 4>와 같은 PDM 데이터베이스 모델을 제품 자료모델로 사용하였다.

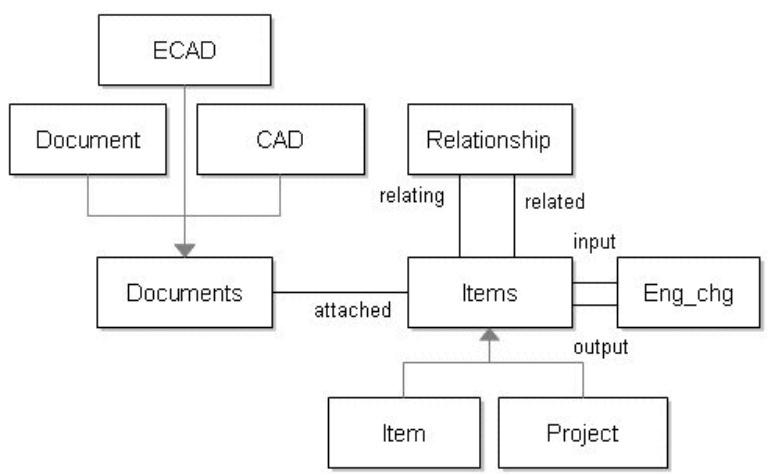

Figure 4. An example of product data model

제안된 제품자료모델은 과제, 제품, 제품구조, 문서, 그리고 설계변경을 표현하고 있으며, <Figure 4>에서 제품의 식별을 통한 부품리스트 구현은 Items의 하위 클래스인 Item 클래스 가, 제품구조는 Relationship 클래스의 related와 relating 속성을 통하여 Item 사이와 Item과 Project 사이의 제품구조를 표현한 다. 설계변경은 Eng_chg 클래스를 이용하여 표현하였다. 설계 변경의 입력과 출력은 각각 input과 output 속성을 통하여 Item 객체를 연결한다. 문서는 Documents 클래스를 이용하여 구현 하였으며, 일반 문서를 뜻하는 Document, 기계 CAD 모델을 뜻 
하는 $\mathrm{CAD}$, 전자 $\mathrm{CAD}$ 모델을 뜻하는 $\mathrm{ECAD}$ 클래스를 하위 클 래스로 가지고 있다. Documents 클래스는 Items 클래스와 연결 되어 해당 제품의 설계정보를 표현한다.

\subsection{PDPE KPI}

PDPE KPI는 PDPE 시스템에서 적용하는 제품개발 성과평 가를 위한 핵심성과지표의 목록이다. 이는 설계 관리자가 수 동으로 확인할 수 있는 목록으로 관리된다. PDPE KPI는 기업 이나 응용 분야에 따라 달라질 수 있으며, 본 논문에서는 문헌 조사를 통하여 PDM 데이터베이스에서 추출할 수 있는 일반적 인 핵심성과지표를 선정하였다.

$<$ Figure 5>는 PDPE KPI를 결정하는 과정을 그림으로 보여 주고 있다. 우선 문헌 조사를 통하여 제품개발 성과평가를 위 한 핵심성과지표(KPI)와 핵심성과지표 구조(KPI Structure)를 조사한다. 특히 본 연구에서는 제품개발 성과평가를 위한 핵 심성과지표를 PDM과 연계한 문헌을 중심으로 조사하였다.

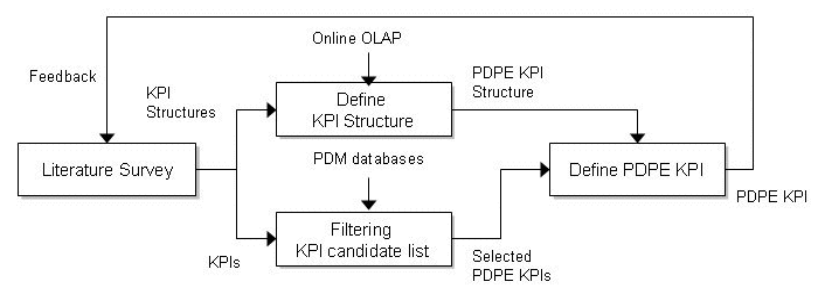

Figure 5. PDPE KPI building process

조사된 자료를 바탕으로 핵심성과지표 구조를 결정한다. 핵 심성과지표 구조란 핵심성과지표의 분류 및 분류 목록 사이의 관계를 뜻한다. 핵심성과지표 구조에 따라 추후 핵심성과지표 들이 해당 분류 목록에 소속되게 된다. 본 연구에서는 핵심성 과지표 전체 체계를 전술/전략과 외부/내부 차원을 조합한 $2 \times 2$ 테이블 형태로 구조화하였다(Tatikonda, 2007). 예로 내부-전술 차원에는 품질, 비용, 그리고 출시시간이 포함되며, 전략-외부 차원에는 새로운 시장 창출과 관련된 핵심성과지표가 포함된 다. 전체 구조에서 핵심성과지표 구조를 결정함에 있어 본 연 구의 목표가 실시간 의사결정 지원 기능이며, PDM 데이터베 이스를 이용하여 핵심지표를 추출하는 점을 고려하였다. PDM 데이터베이스는 설계 정보만 가지고 있으므로 시장이나 고객 관련 정보를 가진 외부와 신기술 개발 등과 관련된 전략적 핵 심성과지표를 온라인으로 제공하는 것이 적합하지 않다. 최종 적으로 PDPE KPI는 전체 핵심성과지표 구조 중에 전술-내부 차원을 지원하는 핵심성과지표만을 제공한다. 아울러 그 분류 는 현재 제품 개발에 가장 핵심적 목표인 품질(Quality), 비용 (Cost), 그리고, 출시시간(Delivery)으로 결정하였다.

$\mathrm{PDM}$ 데이터베이스 기반의 제품개발 성과평가를 위한 핵심 평가지표에 관련된 문헌을 조사하고 이를 바탕으로 약 100 여개 의 핵심성과지표 목록을 확보하였다. 이 목록에는 핵심성과지
표 분류, 이름, 설명, 출처 등의 속성을 가지고 있다. 이들 중에 $\mathrm{PDPE} \mathrm{KPI}$ 를 고르는 과정에서 PDM 데이터베이스를 기반으로 평가지표를 도출하는 제약을 고려하였다. 선택된 핵심성과지 표 구조에 따라 선정된 지표를 구조화함으로써 PDPE KPI가 완 성된다. <Table 1>은 본 연구에서 결정된 PDPE KPI 목록이다.

Table 1. PDPE KPI

\begin{tabular}{l|l}
\hline group & KPI \\
\hline \multirow{5}{*}{ quality } & Q01 : number of products \\
& Q02 : document number per product \\
& Q03 : number of engineering changes \\
& Q04 : number of non-conformal product \\
& Q05 : number of non-conformal drawing (CAD) \\
& Q06 : number of 3D CAD model \\
& Q07 : number of ECAD \\
\hline \multirow{5}{*}{ cost } & C01 : carry-over components percentage \\
& C02 : purchased components percentage \\
& C03 : number of ECO (Engineering Change Order) \\
& C04 : material cost (selling price/material cost) \\
\hline \multirow{5}{*}{ delivery } & D01 : time for engineering change proposal \\
& D02 : time for product approval \\
& D03 : ECO/ECR (Engineering Change Request) \\
& D04 : usage of standard products \\
\hline
\end{tabular}

\section{3 다차원 제품자료모델}

제안된 다차원 제품자료모델은 사실(Fact)로써 제품(Product) 을 선택하고 있다. 여기서 제품이란 최종 제품(End Product) 뿐 만 아니라 이를 구성하는 부품(Parts)까지 포함하는 일반적 개 념이다. 사실의 의사결정 값인 매저(Measure)로써 제품 가격, 중량, 그리고 그 외의 계산된 가치 값을 사용할 수 있다. 본 연 구에서는 1 개의 제품을 사실(Fact)의 단위로 삼았다.

다차원 제품자료모델의 차원(Dimension)으로써는 생성시간 (Time), 생성자(Owner), 기술문서(Document), 제품구조(Product Structure), 그리고 설계변경(Engineering Changes)을 포함한다. 각 차원은 계층(Layer)를 가질 수 있다. 예로 생성시간은 시간, 일, 월, 분기, 그리고 년의 계층을, 생성자는 설계자, 그룹, 부서, 그리고 회사 계층을, 그리고 문서는 CAD 모델, 보고서 등의 계 층을 가질 수 있다. 내용을 단순화하기 위하여 본 논문에서 계 층구조에 대한 서술은 생략한다. <Figure 6>에 제안된 다차원 제품자료모델이 표시되어 있다.

차원 중 제품구조는 Product_structure_related와 Product_structure_relating으로 나누어지며, 설계변경도 Engineering_change input과 Engineering_change_output으로 나누어진다. Product structure related는 해당 제품이 다른 제품의 하위 부품으로써 의 제품구조(관계)를 가지고 있음을 나타낸다. 반면에 Product structure_relating은 해당 제품이 다른 부품의 조립품으로써의 
제품구조를 가지고 있음을 나타낸다. Engineering_change_input 은 해당 제품이 설계변경의 대상 제품임을 Engineering_change output은 해당 제품이 설계 변경의 결과임을 나타낸다 $<$ Figure $6>$ 의 Product_structure_related, Product_structure_relating, Engineering_change_input, 그리고 Engineering_change_output 참조).

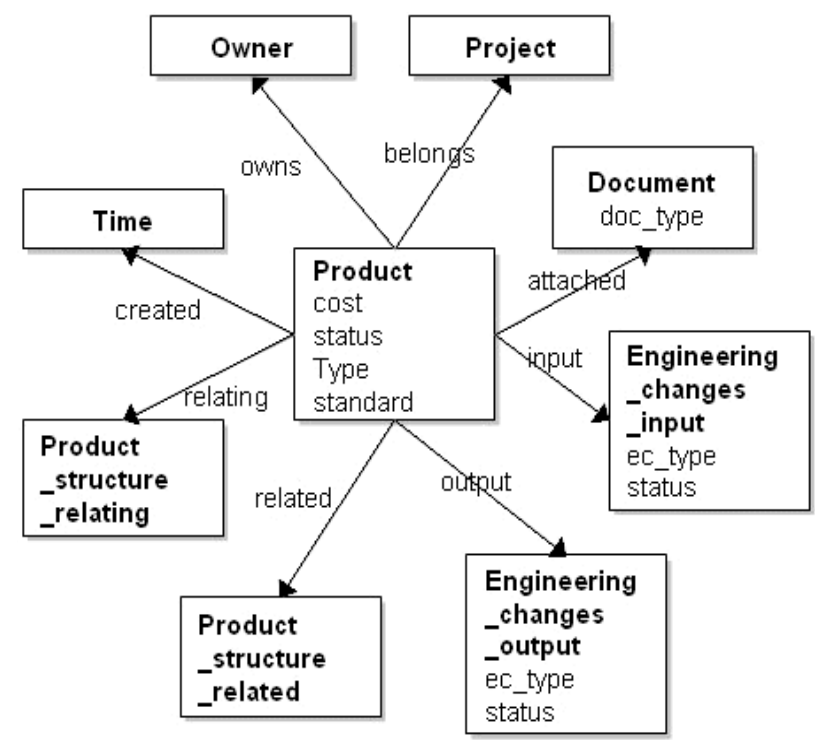

Figure 6. Proposed multidimensional product data model

다차원 제품자료모델은 사실을 매개로 차원 사이의 다양한 관계 조합을 통해 가치 있는 질문을 생성할 수 있다. 예로 과제 별 제품 당 평균 $3 \mathrm{D} \mathrm{CAD}$ 문서 수는 설계 정보가 얼마나 CAD 로 정의되어 있는지를 알 수 있는 성과지표가 될 수 있다. 이 정보는 다차원 제품자료모델의 제품을 중심으로 Document와 Project 차원의 관계를 통하여 필요한 정보를 추출 할 수 있다. 제품에 생성된 문서 중에 doc_type의 속성을 이용하여 3D CAD 문서 개수를 추출하고, 이 제품의 Project 차원의 자료와 연동 시키면 된다. 만일 과제 대신 Time이나 Owner 차원으로 변경 하거나, 이 차원들을 모두 적용한다면 예로 '8월 설계자별 제 품 당 평균 $3 \mathrm{D} \mathrm{CAD}$ 문서 수'와 같은 보다 다양한 조건의 값을 찾아 낼 수 있다.

다른 예로 과제별 표준부품 사용 횟수는 신속한 제품 개발 을 위해 해당 과제에서 표준품을 얼마나 사용하였는지를 알려 주는 중요한 지표이다. 제안된 다차원 제품자료모델에서는 사 실인 Product의 standard 속성을 통한 표준품 여부와 Product structure_related 차원의 횟수를 통하여 추출해 낼 수 있다. 이 경우도 위의 경우와 마찬가지로 Project, Time 그리고 Owner 등 을 이용하면 다양한 측면의 분석 자료를 추출 해 낼 수 있다.

또 다른 예로 설계변경에 대상인 제품의 공유 횟수는 해당 설계변경이 전체 제품구조에 미치는 영향의 크기를 측정할 수 있는 지표이다. 즉 같은 설계변경이라도 변경 대상 제품을 많 은 제품들이 공유한 경우 그 변경의 영향이 커지게 된다. 제안
된 다차원 제품자료모델에서 이 정보는 Engineering_change input과 Product_structure_related 차원을 이용하여 계산할 수 있 으며, 이를 이용하면 단순히 설계변경 대상 제품 수를 측정하 기 위한 Engineering_change_input 차원을 이용한 경우보다 보 다 정교한 정보를 추출할 수 있다.

\subsection{PDPE 데이터웨어하우스와 ETL 모듈}

데이터웨어하우스(Data Warehouse)란 OLAP를 지원하기 위 하여 데이터 큐브 형태로 변화된 자료를 저장하는 독립된 데 이터베이스를 뜻한다. OLAP를 위한 전용 데이터베이스인 데 이터웨어하우스를 OLTP를 위한 운영 데이터베이스(Operational Database)와 분리함으로써 데이터베이스 서버 부하 조정, 요약이나 이력 정보 관리 등의 장점을 가지고 있다. 하지만 분 리된 데이터웨어하우스는 운영 데이터베이스의 자료를 따로 전환하고 부가 정보를 입력해야 하므로 의사결정 자료의 실시 간 처리가 어렵다.

본 연구에서는 사용자의 특별한 개입 없이 PDM 데이터베이 스 자료를 실시간으로 처리하기 위하여 데이터웨어하우스를 데이터베이스 뷰(Database View)와 운영 데이터베이스인 PDM 데이터베이스의 특정 테이블을 공유하는 형식으로 개발하였 다. PDM 데이터베이스에 의사결정을 위한 부가 자료를 넣기 위하여 PDM 응용 프로그램을 확장하여 설계 자료 입력 시 부 가 자료가 해당 테이블에 입력되도록 하였다. 그러므로 PDPE Data Warehouse는 다차원 제품자료모델을 구현하기 위한 PDM 데이터베이스의 특정 테이블과 데이터베이스 뷰의 집합이다.

ETL 모듈(ETL Module)이란 Extract, Transform, 그리고 Load 의 약자로써 운영 데이터베이스와 관련된 여러 데이터베이스 로부터 자료를 추출(Extract)하고 이를 변환(Transform)하여 데 이터웨어하우스로 입력(Load)하는 도구를 뜻한다. 워에서 언 급하였듯이 PDPE 시스템은 데이터베이스 뷰 기능과 PDM 시 스템의 응용 프로그램을 이용하여 PDM 데이터베이스의 자료 를 다차원 제품자료 형태로 실시간 제공하고 있다.

\section{5. 구현}

\section{1 제품설계 자료}

본 연구의 구현을 위하여 자체적으로 개발한 교육용 PDM 시스템을 사용하였다. KEDB TEE(Do, 2012)는 웹 기반 사용자 환경을 제공하는 PDM 시스템으로써 MySQL 데이터베이스 관 리 시스템(MySQL, 2012)과 Java 기반 웹 응용프로그램 개발 도 구를 이용하여 개발되었다. KEDB TEE는 < Figure 4>의 제품자 료모델에 대응되는 자료구조를 제공하고 있으며, 이를 통해 제품설계 자료를 생성한다. <Figure 7>은 제안된 다차원 제품 자료모델을 검증하기 위하여 PDM 시스템을 이용하여 생성된 제품설계 자료의 예이다. 


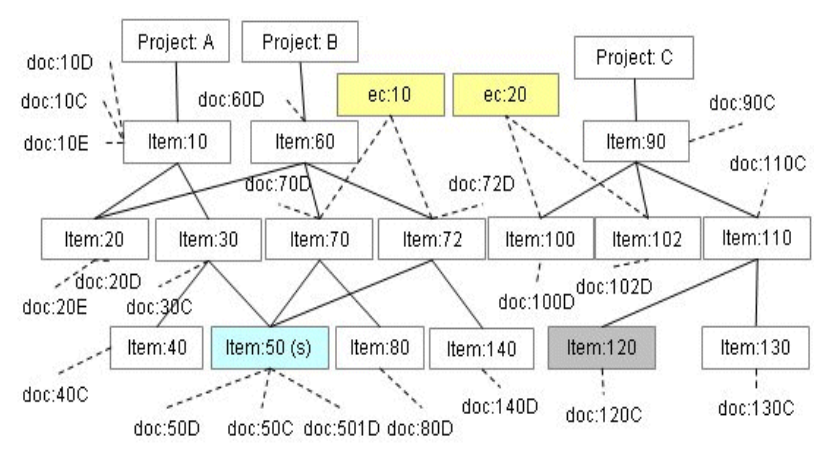

Figure 7. Instantiation example of product design

예에서 총 3개의 프로젝트 $(<$ Figure 7>의 Project : A, Project : $\mathrm{B}$, 그리고 Project : C 참조)가 존재하며 각 프로젝트에는 제품 구조가 존재한다(<Figure 7>에서 Item Instance로 표현된 Item : 10 , Item : 20 등과 이들 사이의 실선 참조). 각 제품은 몇 개의 문서를 가지고 있으며, 이 문서는 일반 문서, CAD 모델, 그리 고 Electrical CAD 모델 등의 종류로 나누어진다. 특정 제품은 다른 제품에 의하여 공유될 수 있으며, 다른 프로젝트에 의하 여 공유될 수 있다(<Figure 7>의 Item : 20은 Item : 10과 Item : 60 에 의하여 공유되며, Project : A와 Project : B에 공유되기도 한다). 예제에서는 두 개의 설계 변경 객체가 존재하며, 각 설 계 변경은 입력과 출력으로 특정 제품과 연결되어 있다(<Figure $7>$ 의 ec : 10 과 ec : 20 Instance 참조).

\section{2 다차원 제품자료모델 구현}

제 4.4절에서 언급한데로 PDPE 시스템은 데이터웨어하우스 를 독립적 데이터베이스로 구현하지 않고 PDM 데이터베이스 의 테이블과 데이터베이스 뷰를 이용하여 구현하였다.

다차원 제품자료모델(<Figure 6> 참조)의 Project 차원은 제 품구조를 검색해야 하므로 관계형 데이터베이스의 뷰로 표현 하기 곤란하였다. 그러므로 Project 차원만 유일하게 PDM 데 이터베이스의 특정 테이블로 구현되었으며, 입력은 제품자료 를 PDM에 입력할 때 프로젝트 자료를 동시에 입력하는 방식 을 취하였다.

Project 외의 다른 차원들은 모두 PDM 시스템의 제품자료모 델에 기반을 둔 데이터베이스 뷰를 통해 구현되었으며, 필요 에 따라 다수의 뷰가 순차적으로 적용되기도 하였다. 예로 Document 차원을 구현하기 위하여 다음의 dim_doc 데이터베이스 뷰가 정의되었다.

CREATE VIEW dim_doc AS SELECT a.oid, b.doc_no, b.doc_ type FROM item a, document $b$ WHERE a.oid = b.item_oid AND a.item_type = 'item';

데이터베이스 뷰 정의에 사용된 SQL(Structured Query Language) 질의에서 item은 제품 정보를 저장한 테이블이며, docu- ment는 문서 정보를 저장한 테이블이다. document 테이블의 문 서가 소속된 제품 식별자를 뜻하는 item_oid 속성을 이용하여 item 테이블과 조인(Join) 을 하여 제품과 연관된 Document 차 원을 구현한 dim_doc 데이터베이스 뷰를 생성하였다.

또 다른 예로 Product_structure_related 차원은 다음 dim_related 데이터베이스 뷰로 구현될 수 있다.

CREATE VIEW dim_related AS SELECT a.oid, b.time_create, a.item_source FROM item a, rel b WHERE a.oid = b.related AND a.item_type = 'item' ;

이 정의에서도 제품구조를 표현하는 rel 테이블과 item 테이 블의 조인을 이용하여 product_structure_related 차원을 구현하 였다. 이때 rel의 부품 식별자를 연결하는 related 속성과 item의 식별자인 oid 속성이 이용된다. 추후 PDPE Client에서 특정 핵 심성과지표를 제공하기 위하여 구현된 차원들을 기반으로 데 이터베이스 뷰와 질의문이 추가된다. <Figure 8>은 MySQL 데 이터베이스 관리시스템 클라이언트 프로그램에서 위에서 언 급한 dim_doc의 내용을 검색한 결과를 보여준다.

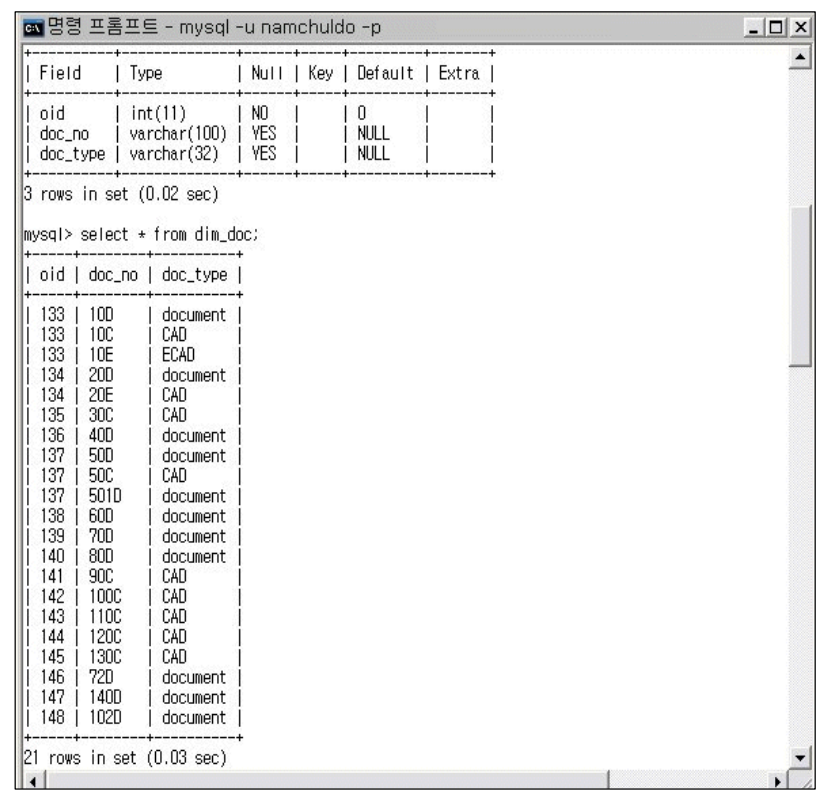

Figure 8. Data type of document dimension and its instances

\section{3 제품개발 핵심성과지표의 질의}

PDPE 클라이언트는 Microsoft 엑셀과 웹 응용 프로그램을 통하여 사용자에게 핵심성과지표를 제공한다. 두 경우 모두 해당 핵심성과지표를 생성하기 위하여 제 5.2절에서 정의된 각 차원에 필요한 데이터베이스 뷰나 질의가 추가되게 된다.

$<$ Figure 9>는 엑셀 클라이언트를 이용하여 핵심성과지표를 출력한 화면을 보여준다. 현재 엑셀 클라이언트는 제품, 문서, 제품구조(공유), 설계변경, 그리고 비용의 5 가지 분류를 통해 
핵심성과지표를 보여주고 있으며, 그림은 문서(Document)에 대한 핵심성과지표와 그래프 출력 화면을 보여준다. 화면에서 는 과제별 문서의 총 수와 제품 당 평균 문서의 수, 그리고 과 제별 $\mathrm{CAD}$ 문서의 총 수와 전체문서 중 $\mathrm{CAD}$ 문서 비율을 보여 주고 있다. 각 핵심성과지표를 출력하기 위해서는 제 5.2절에 서 정의된 Document 차원을 구현한 데이터베이스 뷰에 특정 데이터베이스 뷰와 질의가 추가되게 된다. 이때 뷰의 내용은 엑셀의 $\mathrm{ODBC}$ 데이터베이스 연결을 이용하여 자료를 가져오 며, 질의는 엑셀과 연동된 질의작성기(Microsoft Query)를 통해 구현한다.

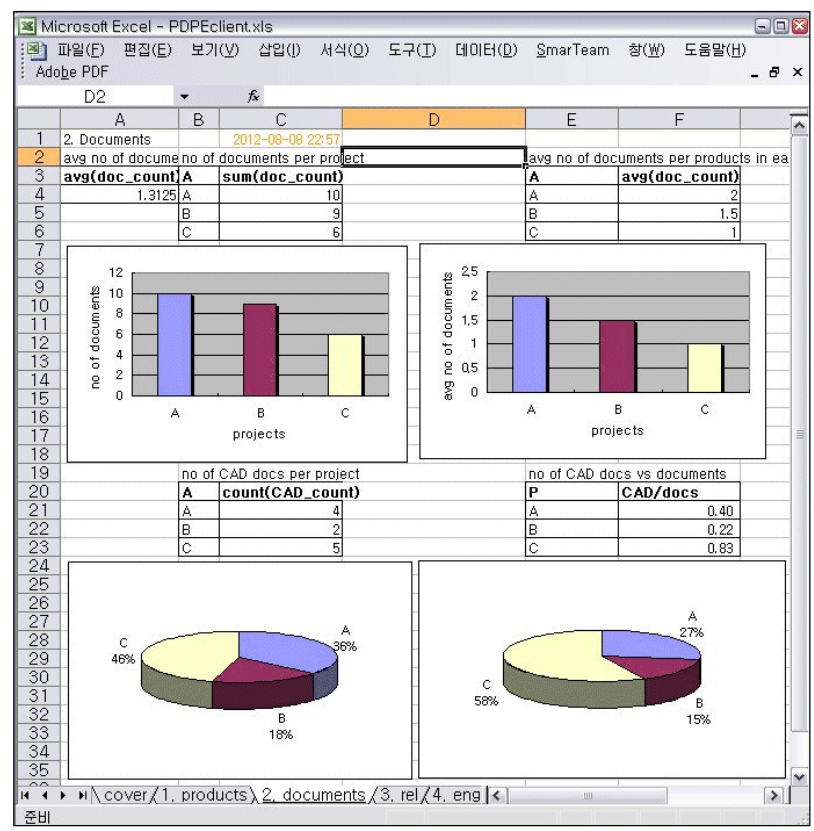

Figure 9. Windows of a PDPE client based on microsoft Excel

엑셀을 이용한 경우 데이터베이스 연결을 통해 가져온 자료 를 가공하여 보다 가치 있는 자료를 만들거나 그래프 등을 통 한 시각화가 편리하다. 예로 < Figure 9>에서 각 과제당 전체 문 서 중 $\mathrm{CAD}$ 문서의 비는 이미 추출된 문서 수량 지표와 $\mathrm{CAD}$ 모 델 수량 지표를 바탕으로 그 비율을 엑셀 상에 수식화하여 표 현하였다. 또한 이 지표의 과제별 차이를 엑셀의 그래프 기능 을 이용하여 쉽게 시각화할 수 있다 $(<$ Figure 9> 우측 하단의 "no of CAD docs vs documents" 항 참조).

다음 cad_count 데이터베이스 뷰 정의는 document 자료 중 $\mathrm{CAD}$ 로만 구성된 차원 자료를 정의한다. 이때 제 5.2절에서 정 의된 dim_doc 데이터베이스 뷰가 기반으로 사용된다.

CREATE VIEW CAD_count AS SELECT oid, COUNT (doc no) 'CAD_count' FROM dim_doc WHERE doc_type = 'CAD' GROUP BY oid;

다음 CAD_count_prj 뷰 정의는 앞의 CAD Document 차원과
Project 차원을 연결시킨다.

CREATE VIEW CAD_count_prj AS SELECT c.item_no, a.oid, a.CAD_count, b.proj_no FROM CAD_count a, dim_proj b, item c WHERE a.oid = b.oid AND a.oid = c.oid;

아래 질의는 앞에서 정의한 뷰로부터 원하는 형태의 정보를 추출하는 역할을 한며, 엑셀에 구현된다.

SELECT proj_no, COUNT(CAD_count) FROM CAD_count prj GROUP BY proj_no;

<Figure 10>은 PDPE 웹 클라이언트를 보여준다. 이 화면은 설계자별 제품과 제품구조의 시간별 누적 생성수를 보여줌으 로써 설계자별 제품 생성 추세를 알 수 있게 해 준다.

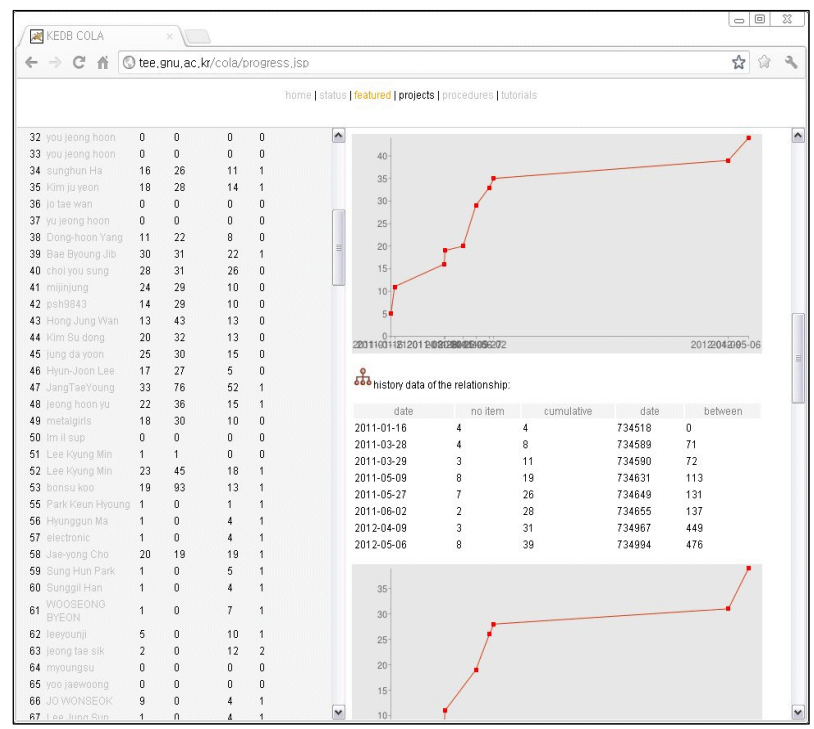

Figure 10. Windows of a PDPE client based on web environment

\section{6. 결 론}

현재 대부분의 기업에서는 일관된 제품정보를 공유하기 위하 여 제품설계 자료의 디지털화가 광범위하게 진행되고 있다. 또한 정보기술의 발전으로 대용량 데이터베이스 기반의 분석 기술이 다양한 분야에 활발히 적용되고 있다. 그러나 PDM 시 스템은 오랫동안 방대한 양의 제품자료를 축적하였음에도 불 구하고, 체계적인 핵심성과지표 추출에 대한 연구 부족으로 축적된 제품자료에 기반을 둔 제품개발 의사결정을 적절히 지 원하지 못하고 있다.

본 연구는 제품자료를 관리하는 PDM 데이터베이스를 기반 으로 제품개발 성과평가에 필요한 다차원 제품자료모델을 고 안하고, 이를 기반으로 실시간 제품개발 성과평가 시스템 아 
키텍처를 제안하였다. 제안된 다차원 제품자료모델을 통하여 $\mathrm{PDM}$ 데이터베이스 자료를 OLAP가 적용가능한 의사결정 지 원 자료로 변경하였다. 제안된 아키텍처의 검증을 위하여 연 구용 PDM 시스템을 운영 데이터베이스로 다차원 제품자료모 델과 OLAP를 구현하였다. 또한 제품개발 성과평가 클라이언 트로써 Microsoft 엑셀과 웹 기반 응용 프로그램을 개발하였다.

시제품과 적용 예제를 통하여 제안된 다차원 제품자료모델 과 제품개발 성과평가 시스템 아키텍처가 PDM 데이터베이스 기반의 실시간 온라인 제품개발 성과평가를 효율적으로 지원 할 수 있음을 확인하였다. 그러므로 제안된 다차원 제품자료 모델을 사용할 경우 광범위하게 사용되고 있는 PDM 데이터베 이스로 부터 제품개발을 평가할 수 있는 실시간 의사결정 지 원 정보를 효과적으로 추출할 수 있게 된다.

본 연구를 기반으로 적용분야와 구현방법 측면에서 추가적 연구를 고려할 수 있다. 제안된 아키텍처는 제품개발 성과평 가를 지원하는 핵심성과지표 추출을 목적으로 하고 있다. 현 재 다차원 제품자료모델은 사실(Fact)의 매저(Measure)로써 횟 수를 사용하고 있는데 이를 대신하여 비용이나 친환경 제품의 주요 가치인 온실가스 발생이력 등을 적용할 경우 다른 의사 결정 분야에 적용이 가능하다.

구현방법 측면에서 현재 시스템은 실시간 온라인 의사결정 지원을 위하여 운영 데이터베이스인 PDM 데이터베이스의 데 이터베이스 뷰와 특정 테이블을 이용하여 데이터웨어하우스 와 ETL 모듈을 구현하였다. 이 구현 방법은 단일 데이터베이 스의 소수의 테이블 조합만으로 관련 KPI을 얻을 수 있기 때문 에, 다차원 제품자료모델을 만들어야 하는 노력에 비하여 그 효용성이 낮을 수 있다. 아울러 서버 부하 분산, 요약과 이력 정보 제공, 그리고 다양한 통합 정보 생성이라는 측면에서 불 리하다. 그러므로 추후 연구로 다수의 데이터베이스를 포함한 이질적 운영 자료를 이용한 OLAP 시스템을 통하여 보다 유연 하고 광범위한 의사결정지원 시스템을 구현할 수 있다.

\section{참고문헌}

Ahmed, Z. (2009), PDM based I-SOAS Data Warehouse Design, proc. 5th International Statistical Conference, 17, 23-25.

Ahmed, Z. (2011), Designing Knowledge Base Towards PDMS, Inter-national Journal of Information Technology and Engineering, 2(1), 9-12.

Alemanni, M., Alessia, G., Tornincasa, S. and Vezzetti, E. (2008), Key performance indicators for PLM benefits evaluation: The Alcatel Alenia Space case study, Computers in Industry, 59, 833-841.

Cheng, Y.-T., Chou, H.-H. and Cheng, C.-H. (2011), Extracting key performance indicators (KPIs) new product development using mind map and Decision-Making and Evaluation Laboratory (DEMATEL) methods, African Journal of Business Management, 5(26), 1073410746.

Do, N. (2007), Introduction to PLM and Its Applications, Saengreung Press, Seoul Korea.

Do, N. (2012), A PLM Introductory Program-KEDB TEE, PLM Best Practice Conference, 21th June, Seoul Korea.

Han, J. and Kamber, M. (2001), Data Mining Concepts and Techniques, Morgan Kaufmann.

MAS-WM (2012), MAS-WM New Product Development (NPD) Gateway, http://www.mas-wm.org/.

MySQL (2012) MySQL, http://www.mysql.com.

Oh, J. and Yang, J. (2010), A Case Study of Implementation of a BSC Performance Evaluation System in Manufacturing Industry based on Product Data Management, IE Interfaces, 23(4), 275-285.

Tan, O. K. and Rasli A. (2011), Prediction of New Product Development (NPD) Performance in Research and Development (R\&D) Company, 3rd International Conference on Advanced Management Science IPEDR, Singapore, 19, 192-200.

Tatikonda, M. V. (2007), Product Development Performance Measurement, chapter in The Handbook of New Product Development, edited by Loch, C. and Kavadias. S., Elesevier Publishers, London, United Kingdom.

Yan, W., Chen, C.-H., Huang, Y., and Mi, W. (2009), A data-mining approach for product conceptualization in a web-based architecture, Computers in Industry, 60, 21-34. 